\title{
Sporotrichosis in an unusual location - Case report*
}

\author{
Brisa Dondoni Reis ${ }^{1}$ \\ Luciana Helena Zacaron ${ }^{1}$ \\ Ricardo Barbosa Lima ${ }^{1}$
}

\author{
Fernanda Oliveira Cobucci ${ }^{1}$ \\ Antonio Macedo D' Acri $^{1}$ \\ Carlos José Martins ${ }^{1}$
}

DOI: http:/ /dx.doi.org/10.1590/abd1806-4841.20153721

\begin{abstract}
A bstract: Sporotrichosis is the most common subcutaneous mycosis. It is caused by the dimorphic fungus Sporothrix schenckii, and the infection is usually acquired by traumatic inoculation. We describe a case of sporotrichosis in an uncommon location with an unusual mode of transmission. A 49-year-old female patient who lived in an urban area of Rio de Janeiro presented with involvement of the left ear. No history of contact with soil, plants or animals was elicited. The suspected source of infection was a pair of handmade wooden earrings. The delay in the diagnosis and treatment resulted in higher morbidity, unsightly scarring and loss of ear lobe.
\end{abstract}

Keywords: Ear; Itraconazole; Mycoses; Sporotrichosis; Wound healing

\section{INTRODUCTION}

Sporotrichosis is a subacute or chronic mycosis caused in most cases by traumatic inoculation of the dimorphic fungus Sporothrix schenckii. ${ }^{1}$

It is the most prevalent subcutaneous mycosis in Latin America, with worldwide distribution. The disease has recently reached epidemic proportions in some regions of Brazil, such as the state of Rio de Janeiro, where the number of cases of zoonotic transmission by infected cats has increased significantly. ${ }^{2}$ The traditional form of transmission, however, is traumatic inoculation of the fungus into the skin, through contact with contaminated soil, plants or organic substrates. $^{3}$

The most common presentation is cutaneous and the disease has been classified into three different clinical forms: cutaneous-lymphatic, fixed and disseminated. ${ }^{4}$
In adults the most common location is the upper limbs, while in children it tends to occur more commonly on the face. ${ }^{4}$

Although the traditional cutaneous-lymphatic form represents most cases of sporotrichosis, the increased incidence of the disease in Brazil has led to an increase in the incidence of atypical and severe clinical forms. ${ }^{5}$

We describe a case of sporotrichosis in an uncommon location (the ear pinna) with an unusual mode of transmission. The delay in the diagnosis and treatment resulted in higher morbidity, unsightly scarring and loss of ear lobe.

\section{CASE REPORT}

A 49-year-old female patient who lived in an urban area of Rio de Janeiro reported a 6-month his-

1 Federal University of the State of Rio de Janeiro (UNIRIO) - Rio de Janeiro (RJ), Brazil. 
tory of symptoms, starting with a pustule in the distal region of the right earlobe, and progressing to an inflammatory, intensely painful lesion affecting the entire pinna. The patient reported previous treatment with systemic antibiotics, oral fluconazole and acyclovir, with no improvement. She denied contact with soil, plants or animals, but reported wearing several pairs of handmade wooden earrings, bought in street markets (Figure 1).

Physical examination revealed erythema and infiltration in the entire right pinna, with exudative areas and crusts. There were floating, erythematous nodules and scar areas in the periauricular region (Figure 2).

Laboratory tests and chest radiography were normal, and serology for HIV was negative.

Histopathological examination revealed a diffuse, granulomatous, chronic inflammatory infiltrate involving the entire dermis (Figure 3). Periodic acid-Schiff (PAS) staining and silver impregnation (Gomori-Grocott staining) showed rounded fungal structures within the inflammatory infiltrate (Figure 4).

A whitish colony with membranous aspect and surrounded by a blackened halo grew on Sabouraud's agar. Microscopy revealed branched, hyaline septate hyphae with oval conidia arranged in a daisy-like pattern at the end of the conidiophore, showing characteristic features of Sporothrix schenckii (Figure 5).

Thus, we concluded that it was a case of cutaneous-lymphatic sporotrichosis.

mg itraconazole per day and were able to observe gradual improvement. Clinical remission occurred in the fifth month. The drug was discontinued after 6 months of therapy (Figure 6).

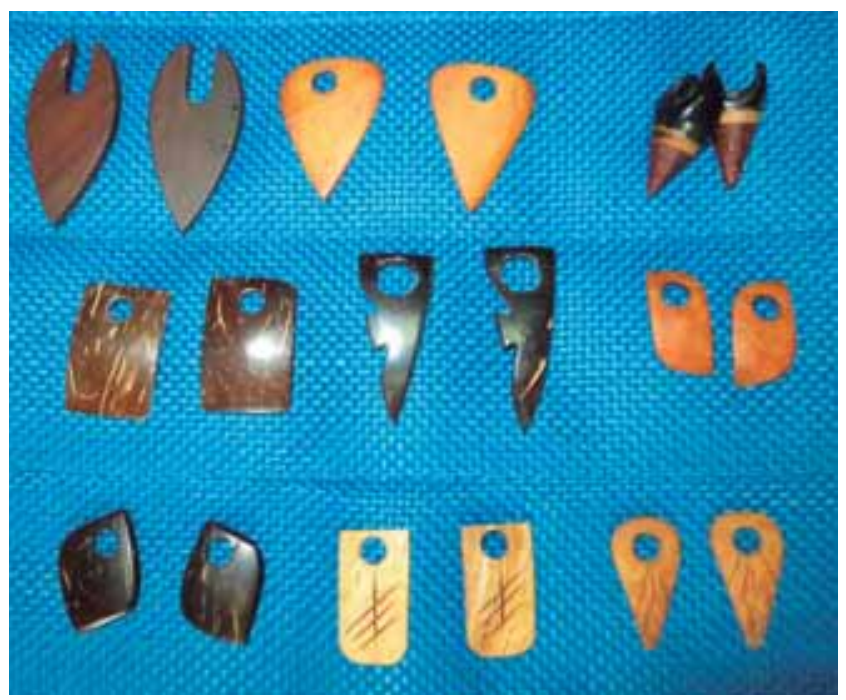

FIGURE 1: Handmade wooden earrings

\section{DISCUSSION}

The sporotrichosis occurs more frequently in workers who deal with contaminated soil and plants. The groups at most risk are gardeners, florists, horticulturists and farmers. However, there have been reports of minor epidemics of sporotrichosis among people who handled straw, hay and bricks. ${ }^{6}$

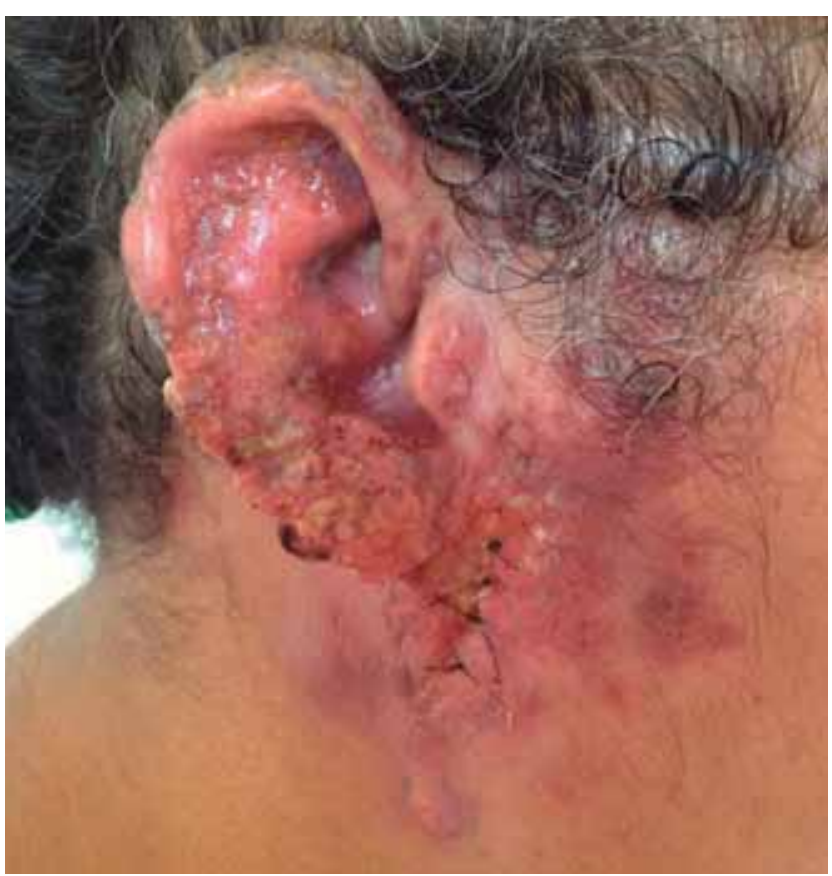

FIGURE 2: Erythema and infiltration in the entire right pinna, with exudative areas and crusts. Floating erythematous nodules and scar areas in the periauricular region

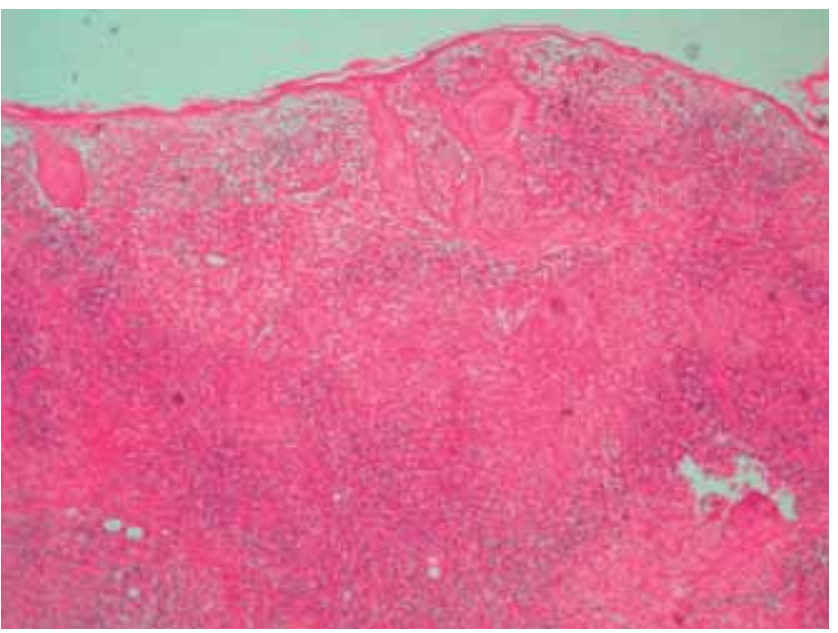

Figure 3: Histopathology showed the presence of a diffuse, granulomatous, chronic inflammatory infiltrate involving the entire dermis $(\mathrm{H} \& \mathrm{E}, 40 \mathrm{x})$ 
The occurrence of the disease is predominantly associated in the literature with professional occupation. It affects persons who deal with soil, particularly in rural areas. ${ }^{1}$ However, since 1998, the city of Rio de Janeiro has been experiencing an epidemic of zoonotic transmission by felines. ${ }^{2}$ The epidemic is due to the presence of the fungus in the environment and to the existence of favorable conditions for maintaining its transmission cycle. ${ }^{1}$

The increased incidence of sporotrichosis in Brazil has led to an increase in the number of cases in unusual locations, with lesions morphologically different from the traditional forms, as well as to the generalization and systematization of the disease. ${ }^{5}$

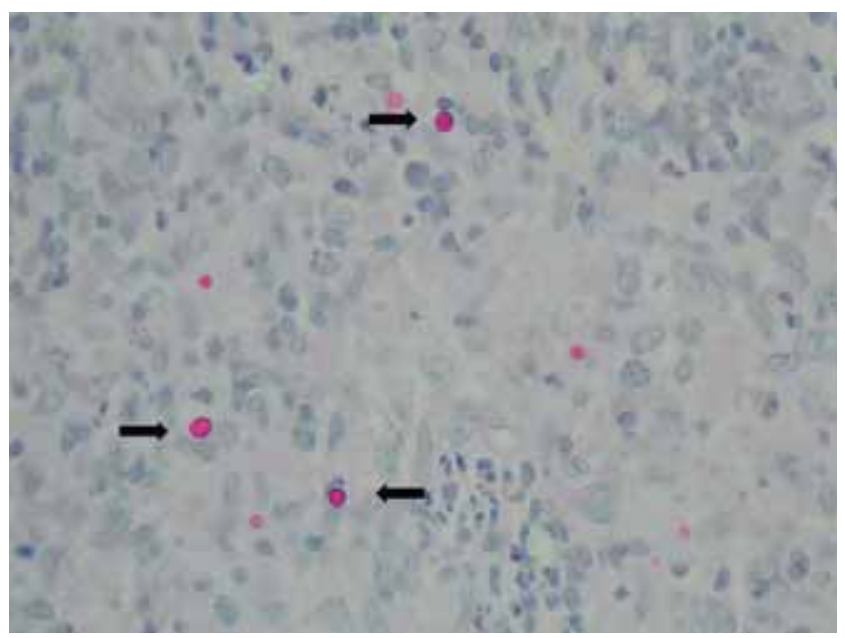

Figure 4: Periodic acid-Schiff (PAS) staining showed rounded fungal structures within the inflammatory infiltrate (400x)
Various clinical presentations are described, however, the localized and cutaneous-lymphatic forms remain the most commonly reported presentations. $^{2}$

The disease mainly affects areas exposed to trauma, such as the extremities and the face. ${ }^{6}$ In children, the most common location is the face, whereas in adults it tends to occur most commonly on the hands, forearms and arms. ${ }^{4}$

Our literature review revealed only two cases of sporotrichosis affecting the pinna.

The first case was described in 1979 in Denver, Colorado, on a 31-year-old mason. The suspected mode of transmission was contact with bricks. The possibility of this type of infection has been previously reported three times in the literature. The mason was initially treated with oral and intravenous antibiotics, without success. After diagnosis, a therapy with potassium iodide was started and the wound healed.?

The second case was published in 1985 in the Brazilian Annals of Dermatology on a 8-year-old boy from São Borja, Rio Grande do Sul. The mode of transmission was not mentioned. However, the authors stressed the difficulty of diagnosing the disease, which was initially treated as pyoderma. ${ }^{8}$

In our case, as the patient reported no contact with soil, plants and animals, we considered the wearing of contaminated wooden earrings as the mode of inoculation of the fungus into the patient's ear lobe, where the lesions started.

The uncommon location and the absence of the usual epidemiological history caused a delay in diagnosis and proper treatment, thus increasing morbidity and resulting in the partial loss of the patient's ear lobe.

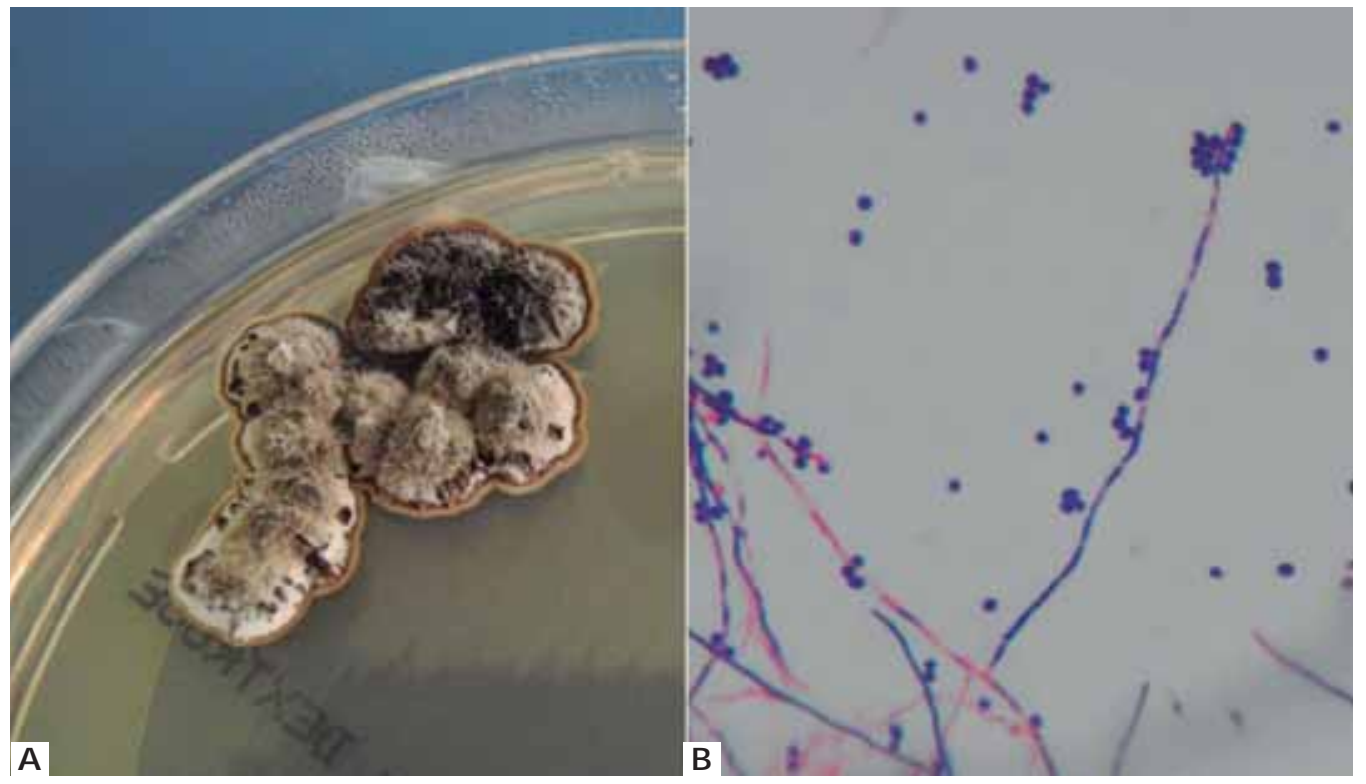

\section{Figure 5:}

A) A whitish colony with membranous aspect and surrounded by a blackened halo is seen in the Sabouraud's agar macroculture. B) Microscopy reveals branched, hyaline septate hyphae with oval conidia arranged in a daisy-like pattern at the end of the conidiophore, showing characteristic features of Sporothrix sp (PAS, 400x) 

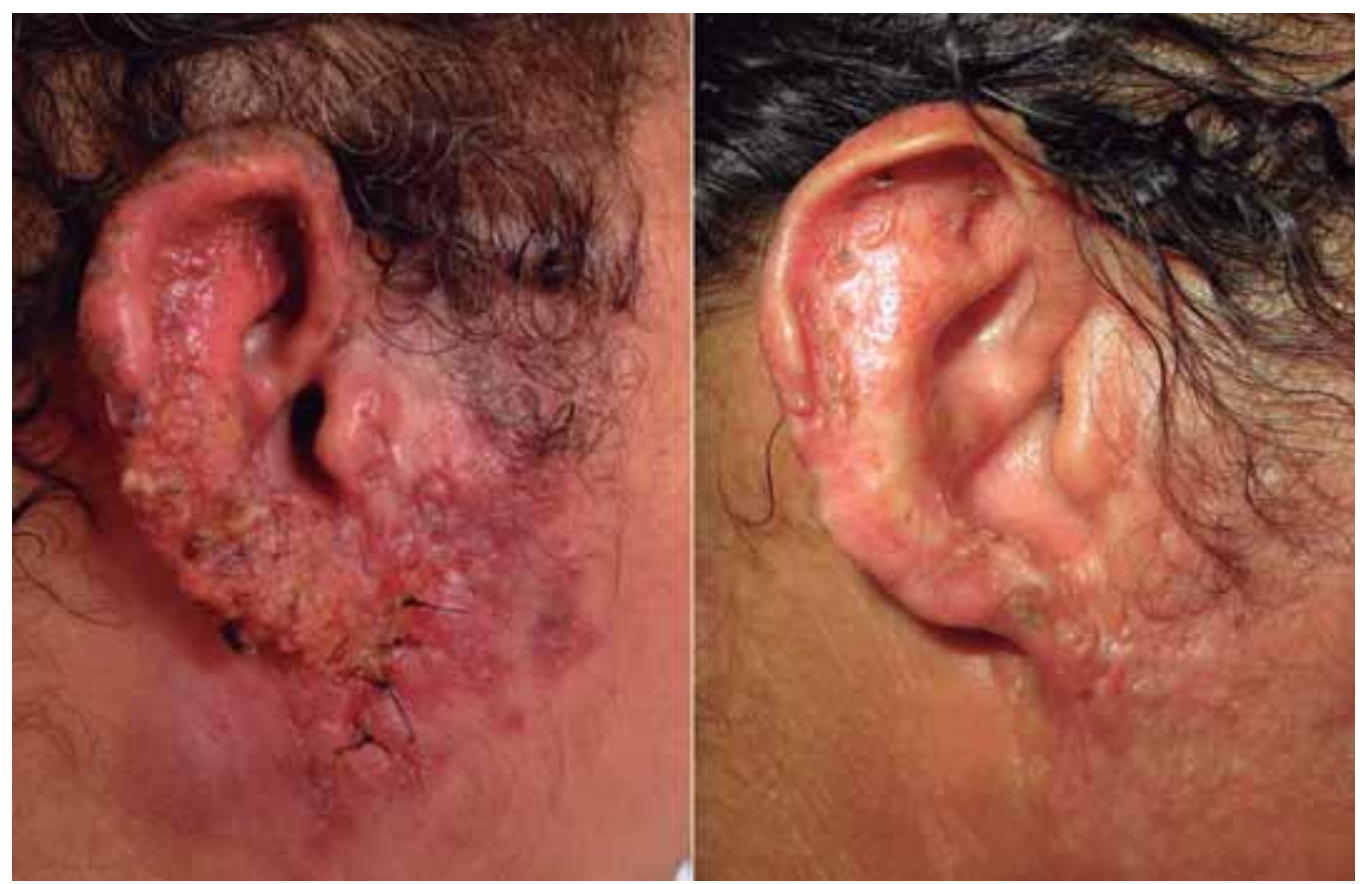

\section{Figure 6:}

Result observed in the fifth month of treatment

An uncommon aspect that contributed to the diagnosis in our case was the finding of fungal structures in the histopathological examination, as these structures are rarely observed histopathologically. ${ }^{9}$

Sporotrichosis is usually a chronic disease with great polymorphism. It may take several clinical forms. A high clinical suspicion and a detailed history are essential for diagnosis. ${ }^{6}$ The lesional polymorphism of sporotrichosis makes the diagnosis on clinical grounds difficult. Therefore, it is of paramount importance to clinically suspect the mycosis even in unusual locations such as the ear pinna. ${ }^{8}$ Because sporotrichosis is considered an endemic disease in Brazil and has several clinical presentations, it should always be listed among the possible diagnoses.

The aim of this report of a case of sporotrichosis in an uncommon location with an unusual mode of inoculation is to illustrate another form of presentation of this mycosis, in order to contribute to early diagnosis and treatment, and reduce morbidity and chronicity of the disease. $\square$

\section{REFERENCES}

1. Silva MB, Costa MM, Torres CC, Galhardo MC, Valle AC, Magalhães Mde A, et al. Esporotricose urbana: epidemia negligenciada no Rio de Janeiro, Brasil/ Urban sporotrichosis: a neglected epidemic in Rio de Janeiro, Brazil. Cad Saude Publica. 2012;28:1867-80.

2. Cordeiro FN, Bruno CB, Paula CD, Motta Jde 0. Familial occurrence of zoonotic sporotrichosis. An Bras Dermatol. 2011;86:S121-4.

3. de Lima Barros MB, de Oliveira Schubach A, Galhardo MC, Schubach TM, dos Reis RS, Conceição MJ, et al. Sporotrichosis with widespread cutaneous lesions: report of 24 cases related to transmission by domestic cats in Rio de Janeiro, Brazil. Int J Dermatol. 2003;42:677-81.

4. Vásquez-del-Mercado E, Arenas R, Padilla-Desgarenes C. Sporotrichosis. Clin Dermatol. 2012;30:437-43.

5. Schechtman RC, Crignis GS, Pockstaller MP, Azulay-Abulafia L, Quintella LP, Belo M. Molluscum-like lesions in a patient with sporotrichosis. An Bras Dermatol. 2011;86:1217-9

6. Neto Rda J, Machado AA, de Castro G, Quaglio AS, Martinez R. Disseminated cutaneous sporotrichosis as the initial manifestation of acquired immunodeficiency syndrome--case report. Rev Soc Bras Med Trop. 1999;32:57-61.
7. Cox RL, Reller LB. Auricular Sporotrichosis in a Brick Mason. Arch Dermatol. 1979;115:1229-30.

8. Severo LC, Gutierrez MJ. Esporotricose auricular em criança. An Bras Dermatoo 1985;60:347-8.

9. Heidrich D, Stopiglia CD, Senter L, Vetoratto G, Valente P, Scroferneker ML. Successful treatment of terbinafine in a case of sporotrichosis. An Bras Dermatol. 2011;86:S182-5

M AILING ADDRESS:

Brisa D ondoni Reis

Rua M ariz e Barros, 775

20.270-004 -Rio de Janeiro - RJ

Brazil

E-mail: brisadondoni@hotmail.com

How to cite this article: Reis BD, Cobucci FO, Zacaron LH, D'Acri, AM, Lima RB, Martins CJ. Sporotrichosis in an unusual location: a case report. An Bras Dermatol. 2015;90 (3 Suppl 1): S84-7. 